\title{
Incorporating the size principle into an ensemble of back-propagating neurons
}

\author{
STEPHEN J. GOTTS \\ The Psychological Corporation, San Antonio, Texas \\ and \\ FREDERICK J. BREMNER \\ Trinity University, San Antonio, Texas
}

\begin{abstract}
As researchers try to move from cybernetics to neural reality, it is time to look at the match between the back propagation strategy and the functional parameters of neurons. Traditionally, the "bias value" in the back propagation activation function has served a mathematical rather than a biological function. By incorporating a production system into the activation logic at the hidden layer, we are able to arrive at a conditional bias value that approximates the function of thresholds in biological neurons.
\end{abstract}

The field of neural networks has made much progress since the advent of the perceptron (Rosenblatt, 1958). The perceptron model had little correspondence to biological neurons either at the level of the single neuron or at the level of the network (Gardner, 1993). It also had a limited range of application since it was unable to learn even simple nonlinear relationships such as the exclusive-or rule (Minsky \& Papert, 1969). Back propagation, a multilayered perceptron armed with the delta learning rule, solved the perceptron's inability to learn nonlinear patterns (Rumelhart \& McClelland, 1986). However, it still has many critics in the biological and social sciences (Crick, 1989; Lockery \& Sejnowski, 1993; Selverston, 1988). The nature in which it propagates its error during learning has little biological support, and its lack of single-neuron properties causes many to be skeptical that any useful analogy to nervous system functioning is possible (Gardner, 1993). These criticisms certainly have merit, and all those who use back propagation to gain insight into the functioning of the brain should take heed. It is possible to generalize from the back propagation network to the brain only to the extent that the two function similarly. If there is little similarity to brain functioning, there can be little confidence in any inferences made about brain functioning.

In our effort to move back propagation closer to the functioning of biological neurons, we have chosen to model the relationship between vertebrate motoneurons and the skeletal muscle fibers with which they innervate. Inputs to this system of various speeds recruit motoneurons of various sizes to initiate a muscle contraction (Henneman, 1980). Motoneurons with small cell bodies have lower activation thresholds than do larger motoneurons, causing them to be relatively more excitable (Carew \& Ghez, 1985). Slow efferent input stimulates only these smaller motoneurons be-

Correspondence should be addressed to S. J. Gotts, 1250 E. Mulberry 218, San Antonio, TX 78209 (e-mail: steven_gotts@hbtpc.com). cause the current is not positive enough to overcome the thresholds of the larger motoneurons. However, as the input becomes faster, the current becomes more positive and larger motoneurons become involved in stimulating the muscle. This has come to be known as the "size principle" of motoneurons (Henneman, 1980).

In order to model the size principle with success, one must introduce variable soma sizes and variable threshold values into the standard back propagation network. This will be accomplished in two steps, the first of which is to add production system logic to the hidden layer of the network. A production statement will allow each hidden neuron to determine if the input frequency is sufficient to overcome its particular threshold. If the hidden neuron is assigned a small soma size, lower input frequencies will be required for it to propagate activation than if it had been assigned a larger soma size. The second step is to use the "bias value" either to boost hidden neuron activation frequency to a certain degree once a threshold is overcome or to remove activation frequency if the input frequency is too low. This is somewhat of a departure from the standard uses of the bias value; as previously, it has been used to generate at least minimal output when input is too low (Fetz, 1993) or to stabilize and enhance network learning (Baxter \& Byrne, 1993; Leshno, Lin, Pinkus, \& Schocken, 1993; Mptitsos, Burton, \& Creech, 1988).

Using this two-fold strategy, we predict that we will be able to recruit hidden neurons of the appropriate size for efferent inputs of different speeds. Additionally, we will compare the learning and error rates of this new, more neuromorphic network with those of a standard back propagation network at several different levels of convergence.

\section{METHOD}

\section{Apparatus \\ A 4-Mb Compudyne (IBM-compatible) 486DX personal com- puter operating at $33 \mathrm{mHz}$ was used to implement the simulation.}


The standard and altered back propagation neural networks were written in Microsoft C/C++ 7.0 (Microsoft Corp., 1991).

\section{Input Layers}

The input layers of the standard back propagation network and the altered back propagation networks were identical and consisted of a single input neuron. The input neuron symbolized the efferent input to the motoneurons, which are represented by the hidden layer. Values at this layer were scaled between 0 and 1 to facilitate learning. An input value can be conceptualized as the ratio of current frequency to the fastest frequency possible. For example, an input of .3 would indicate that the input neuron was firing at $30 \%$ of its potential rate.

\section{Hidden Layers}

The hidden layer of the standard back propagation network consisted of 10 hidden neurons with no alterations to the activation function. The hidden layer of the altered, more neuromorphic back propagation network consisted of 10 hidden motoneurons, each assigned a different soma size between 0 and 1 (Figure 1). The sizes were distributed proportionally to the gastrocnemius motoneuron pool described in Henneman (1980). As the relationship between soma size and threshold was assumed to be one to one, the threshold value for each motoneuron is identical to its soma size. For each hidden motoneuron, a simple production statement was used to determine whether the input frequency was greater than the threshold value. If the threshold was overcome, the raw activation was computed as follows:

$$
\text { raw activation }=\left(\text { input } * w_{j i}\right)+(\text { bias } * \text { threshold }),
$$

where $w_{j i}$ is the connection strength between the input and hidden neurons, and bias is a constant set to be 4.8485 . This bias value resulted in an average (bias $*$ threshold) value of 2.0 . If the threshold was not overcome by the input frequency, the raw activation was computed as follows:

$$
\text { raw activation }=\left(\text { input } * w_{j i}\right)-\text { bias' }^{\prime}
$$

where bias' was a constant set to be 2.0 . This approach was taken to assure that, on average, the same amount of activation would be added or subtracted. Also, if a particular hidden neuron's threshold was overcome, it would propagate the appropriate amount of activation according to its size and assumed conduction velocity, as a large neuron should have a faster conduction velocity and higher hidden raw activation than would a small neuron (Henneman, 1980). Once raw activation was computed for all hidden neurons, the standard sigmoid activation function was used to "squash" raw activation between 0 and 1 . A single hidden neuron's squashed activation represented the firing frequency of a motoneuron.

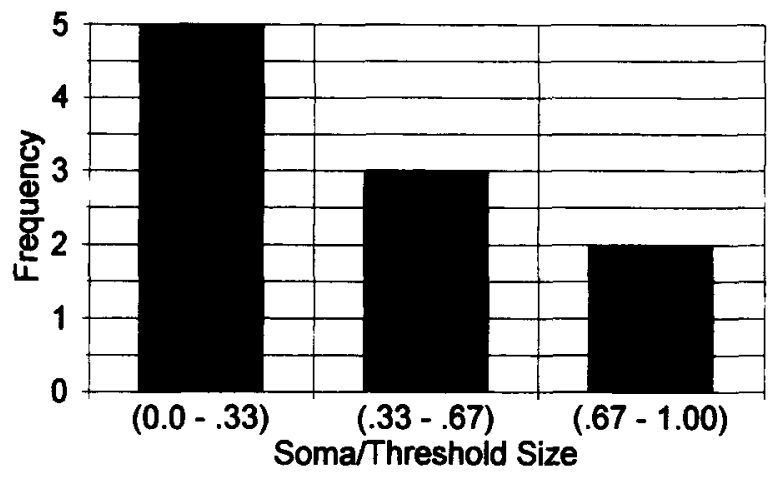

Figure 1. Frequency distribution of artificial soma/threshold sizes of back propagation motoneurons at the hidden layer. The distribution is modeled after the motoneuron pool for the gastrocnemius muscle in rhesus monkeys (Henneman, 1980).

\section{Output Layers}

The output layers of the standard and altered back propagation neural networks were identical and consisted of a single output neuron. The output node represented overall tetanic tension in a gastrocnemius muscle. As in the input layer, values were scaled between 0 and 1 . A single output value was the ratio of current tetanic tension to total possible tetanic tension.

\section{Procedure}

The two different types of 1-10-1 (number of neurons at the input, hidden, and output layers, respectively) networks, standard back propagation, and altered back propagation were assigned random connection strengths (or "weights") 30 different times for each convergence level tested. In using 30 different randomizations, we were confident that any observed effect was not the result of a single random event. Each network was applied to the same set of 25 inputoutput training facts representing the identity function. The identity function $(y=x)$ was chosen because the relationship between efferent input frequency and output tetanic tension was assumed to be direct and one to one. Networks were allowed to self-organize until the entire input set was mapped to its corresponding output set below a specified level of convergence. The number of training iterations was recorded for each of the networks at each level of convergence.

A set of 25 new input-output patterns also representing the identity function was presented to each network to evaluate how each trained network responded to patterns it had never encountered. This phase was designed to provide evidence that the networks had not overlearned the specific input -output patterns in the training set. For each network at each convergence level, the number of testing facts eliciting output error within the convergence level was recorded, as well as the average output error across the 25 testing facts and hidden motoneuron activation frequencies for each testing fact.

\section{RESULTS AND DISCUSSION}

A visual analysis of the Hinton diagrams (Bremner, Gotts, \& Denham, 1994; Hinton \& Shallice, 1991) for the altered back propagation network revealed that hidden motoneurons were indeed being recruited correctly according to soma size and input frequency. Figure 2 shows a Hinton diagram of the hidden layer activations for one of the 30 weight randomizations trained at the 1 level of error convergence. Hidden neurons have been arranged from 1 to 10 -smallest to largest soma size. As input frequency increases, larger motoneurons join the group of neurons that are active in contracting the gastrocnemius muscle. Notice, also, that bigger motoneurons are more active than are smaller motoneurons once recruited.

Number of training iterations necessary for convergence was compared for both the standard and altered back propagation networks. $T$ tests revealed that for both the .1 and .095 levels of convergence $(N=30$ for each comparison), the standard network trained significantly faster than did the altered network $(p<.01$ for both levels). However, for the $.085, .08$, and .075 levels of convergence $(N=30$ for each comparison), the altered network trained significantly faster than did the standard network ( $p<.01$ for each level). Mean number of necessary training iterations for both network types at each level of convergence is provided in Figure 3. For the standard back propagation network with no thresholds, number of necessary training iterations escalated rapidly from 


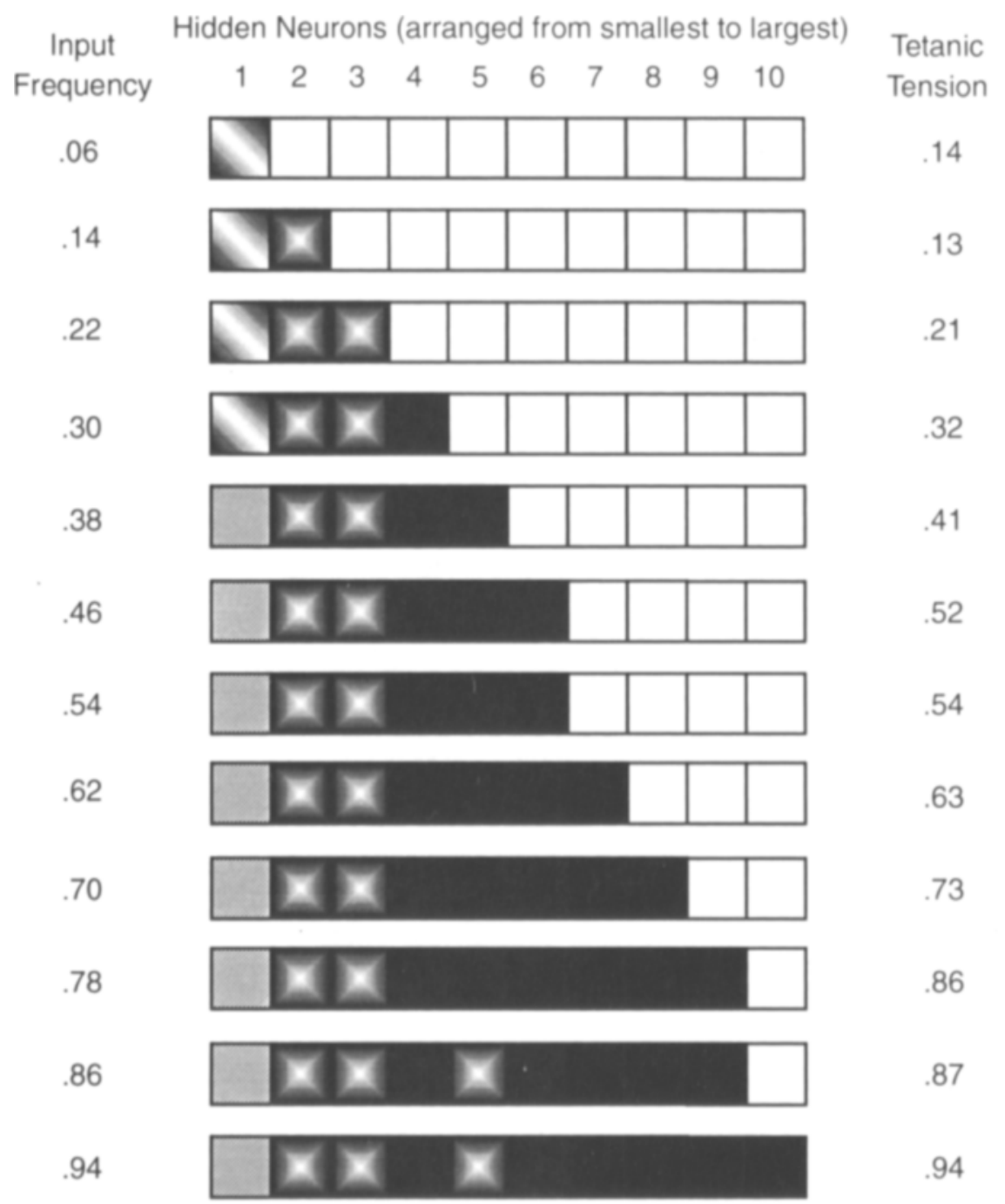

Hidden Activation Frequency:

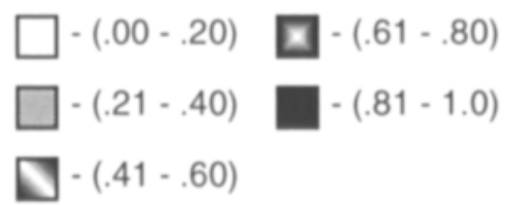

Figure 2. A Hinton diagram (Bremner, Gotts, \& Denham, 1994; Hinton \& Shallice, 1991) of the hidden layer activation frequencies for 12 test stimuli of increasing frequency. Hidden neurons 1 through 10 are arranged in order of ascending soma/ threshold size. The darker a particular node is shaded, the higher is its activation frequency.

the .08 to the .075 levels. The standard network failed to converge at the .07 level, while the altered back propagation network continued training until the .03 level of convergence $(M=3351.066, S E=109.605, N=30)$.

Average output error across the 25 testing facts was compared for both the standard and altered back propagation networks at each level of convergence. $T$ tests revealed that for all levels of convergence $(N=30$ for each comparison), the standard back propagation network had significantly lower average output error than did the altered network ( $p<.05$ for the .1 level, $p<.01$ for the $.095, .09, .085, .08$, and .075 levels). Figure 4 shows the output error averaged across the 25 testing facts and the 30 network randomizations for both types of networks at each level of convergence. While it is true that the standard back propagation network was more accurate than was the altered network for all the levels at which it was able to converge, the altered network ultimately became the 


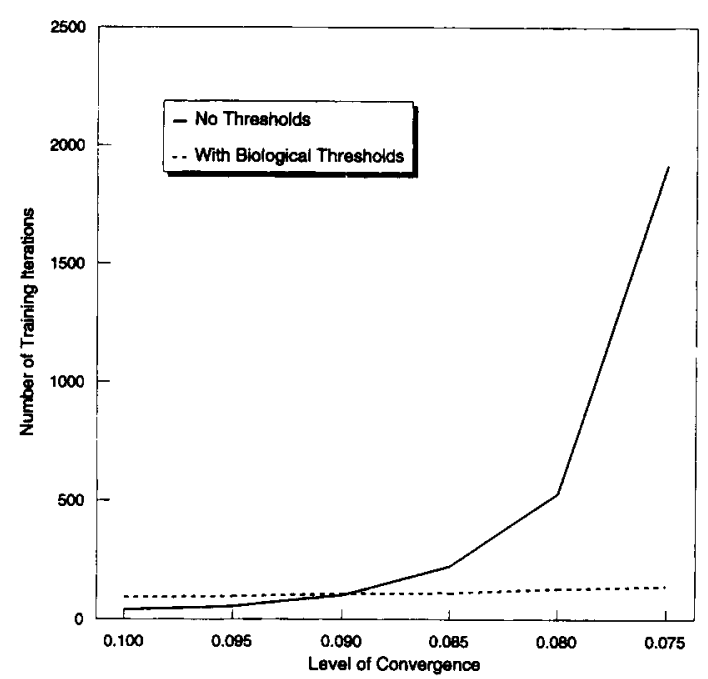

Figure 3. A diagram depicting the relationship between level of convergence and number of necessary training iterations. Thirty networks were trained for both standard back propagation networks and altered networks at each level of convergence; the average of each group of $\mathbf{3 0}$ networks is depicted at each level of convergence for both types of networks. Standard networks are described by a solid line, while the altered networks are described by a dashed line.

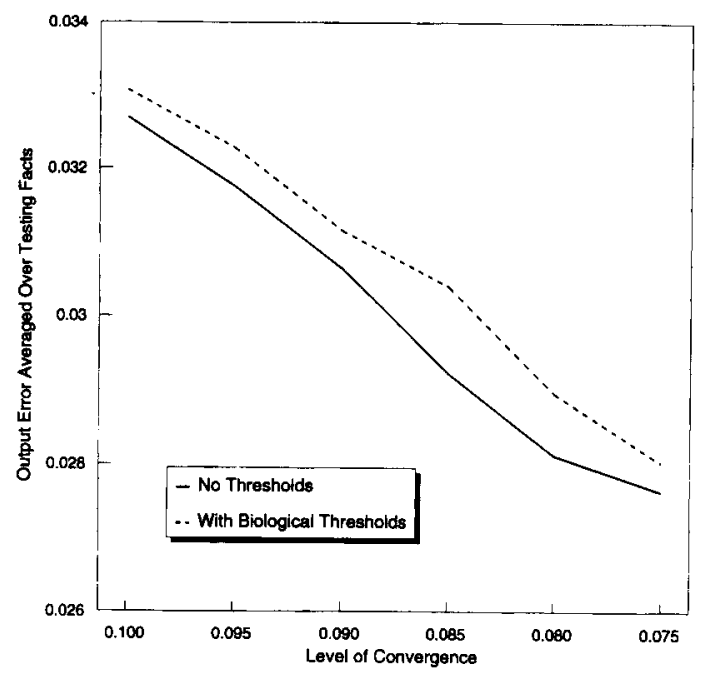

Figure 4. A diagram of output error averaged across the 25 testing facts as a function of level of convergence. Thirty networks were trained for both standard back propagation networks and altered networks at each level of convergence; the average of each group of 30 networks is depicted at each level of convergence for both types of networks. Standard networks are described by a solid line, while the altered networks are described by a dashed line.

more accurate as it reached the .03 level of convergence $(M=.00973, S E=.00003, N=30)$.

Clearly, there are numerous benefits from adding the more "biological" thresholds at the hidden layer. The hidden neurons are selected according to the size principle, as predicted, establishing that we have moved our back propagation model toward a more neuromorphic state. Addi- tionally, the thresholds dramatically reduced necessary training time for lower levels of convergence and ultimately led to more accurate predictions. Every altered network generated output error within the desired convergence level for at least 24 out of 25 of the testing facts. While the standard back propagation network seems to perform more accurately for levels at which it is able to converge, the training times for lower levels of convergence are extremely costly, and its ability to converge at very low levels is sharply limited.

Thus, with a few simple alterations, we have made a back propagation neural network function more like a biological network. It should follow that by doing so, we have improved the analogy between back propagation functioning and central nervous system functioning. While higher cognitive systems may need different changes in back propagation to improve neuromorphology, we have demonstrated the efficacy of such changes in a simple neural system.

\section{REFERENCES}

BAXTER, D. A., \& BYRnE, J. H. (1993). Learning rules from neurobiology. In D. Gardner (Ed.), The neurobiology of neural networks (pp. 71105). Cambridge, MA: MIT Press.

Bremner, F. J., GotTs, S. J., \& Denham, D. L. (1994). Hinton diagrams: Viewing connection strengths in neural networks. Behavior Research Methods, Instruments, \& Computers, 26, 215-218.

CAREW, T. J., \& GHEZ, C. (1985). Muscles and muscle receptors. In E. R. Kandel \& J. H. Schwartz (Eds.), Principles of neural science (2nd ed., pp. 443-456). New York: Elsevier.

Crick, F. (1989). The recent excitement about neural networks. Nature, 337, 129-132.

FETZ, E. E. (1993). Dynamic neural network models of sensorimotor behavior. In D. Gardner (Ed.), The neurobiology of neural networks (pp. 165-190). Cambridge, MA: MIT Press.

GARDNER, D. (1993). Introduction: Toward neural neural networks. In D. Gardner (Ed.), The neurobiology of neural networks (pp. 1-11). Cambridge, MA: MIT Press.

Henneman, E. (1980). Organization of the motoneuron pool. In V. B. Mountcastle (Ed.), Medical physiology: Vol. 1 (14th ed., pp. 718741). St. Louis: Mosby.

Hinton, G. E., \& SHAllice, T. (1991). Lesioning an attractor network: Investigations of acquired dyslexia. Psychological Review, 98, 74-92.

Leshno, M., Lin, V. Y., Pinkus, A., \& Schocken, S. (1993). Multilayer feedforward networks with a nonpolynomial activation function can approximate any function. Neural Networks, 6, 861-867.

LOCKERY, S. R., \& SEJNOWSKI, T. J. (1993). Realistic network models of distributed processing in the leech. In D. Gardner (Ed.), The neurobiology of neural networks (pp. 107-135). Cambridge, MA: MIT Press.

Microsoft CorP. (1991). Microsoft C/C ++ (Version 7.0) [Computer program]. Redmond, WA: Author.

Minsky, M. L., \& PAPERT, S. A. (1969). Perceptrons: An introduction to computational geometry. Cambridge, MA: MIT Press.

Mpitsos, G. J., Burton, R. M., \& Creech, H. C. (1988). Connectionist networks learn to transmit chaos. Brain Research, 21, 539-546.

RosenblatT, F. (1958). The perceptron: A probabilistic model for information storage and organization in the brain. Psychological Review, 65, 386-408.

Rumelhart, D. E., \& McClelland, J. L. (Eds.) (1986). Parallel distributed processing: Explorations in the microstructure of cognition: Vol. 1. Foundations. Cambridge, MA: MIT Press.

SELVERSTON, A. I. (1988). A consideration of invertebrate central pattern generators as computational data bases. Neural Networks, 1, 109-117.

(Manuscript received December 4, 1995; accepted for publication January 3, 1996.) 